\title{
Successful TAMIS in an elderly patient with a large rectal adenoma
}

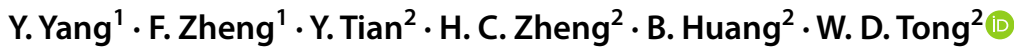 \\ Received: 29 January 2021 / Accepted: 29 May 2021 / Published online: 10 July 2021 \\ (c) Springer Nature Switzerland AG 2021
}

Endoscopic local resection is currently recommended for rectal adenoma and highly selected $\mathrm{T} 1$ rectal cancers without lymph node invasion. However, local resection under colonoscopy is always difficult for huge rectal villous adenomas, even using transanal minimally invasive surgery (TAMIS) that has emerged in recent years. We successfully excised a large rectal adenoma using TAMIS with preservation of the muscular layer of the rectum.

The patient was an 80 -year-old man with a $7 \times 6 \times 1 \mathrm{~cm}^{3}$ soft and broad-based tubulovillous adenoma located on the anterior wall of the rectum, at 5-12 cm from the anal verge. Magnetic resonance imaging revealed that the rectal lesion was confined to the mucosa and submucosa.

The surgical procedure is shown in the attached video. The patient was under general anesthesia in the left lateral position. The rectum and anus were washed and sterilized with iodophor. The single port access system (STAR port, Shinaide, Xiamen, China) was placed in the anus. Pneumoperitoneum was established to maintain a constant pressure level at $12 \mathrm{mmHg}$. The lesion was exposed under the highdefinition display of the laparoscope. The cutting edge was labeled with an electrocoagulation hook $(0.5-1.0 \mathrm{~cm}$ away from the tumor edge), followed by accurate submucosal complete resection along the preset surgical margin line, with the intact muscle layer of the rectal annulus retained, while the wound surface was too large to suture the mucosa. On histological examination both the vertical and horizontal margins were negative.

The postoperative course was uneventful. Follow-up examination 1 and 3 months after the operation showed good recovery.

Supplementary Information The online version contains supplementary material available at https://doi.org/10.1007/s10151-021-02474-z.

\section{Declarations}

Conflict of interest The authors declare that they have no conflict of interest.

Ethical approval The patient was managed as per the standard guidelines and institutional treatment protocols.

Informed consent Informed consent was obtained from the patient for the publication.

Publisher's Note Springer Nature remains neutral with regard to jurisdictional claims in published maps and institutional affiliations.

Yong Yang and Fan Zheng contributed equally to the work and are co-first authors.

\footnotetext{
W. D. Tong

vdtong@163.com

1 Department of Gastrointestinal Surgery, People's Hospital of Deyang City, Deyang, Sichuan Province, China

2 Department of General Surgery, Army Medical Center (Daping Hospital), Army Medical University, Chongqing, China
} 